|| ISSN(online): 2589-8698 || ISSN(print): 2589-868X || International Journal of Medical and Biomedical Studies

Available Online at www.ijmbs.info

Volume 3, Issue 2; February: 2019; Page No. 30-39

PubMed (National Library of Medicine ID: 101738825)

Index Copernicus Value 2017: 40.03

\title{
PREVALENCE OF SYPHILIS AMONG PREGNANT WOMEN ATTENDING ANTENATAL CLINIC OF BULCHANA HEALTH CENTER, SHASHEMENE, WEST ARSI ZONE, ETHIOPIA
}

\author{
Menberu Wubete ${ }^{1}$, Musa Mohammed ${ }^{2}$, Mulugeta Mengistu $^{3}$, Robel Mekonnen ${ }^{4}$ \\ ${ }^{1}$ BSc., Department of Medical Laboratory Sciences, College of Medicine and Health Sciences, Dire Dawa \\ University, Dire Dawa, Ethiopia. \\ ${ }^{2}$ BSc., MSc., PhD. Candidates in Medical Microbiology, Department of Medical Laboratory Sciences, \\ College of Medicine and Health Sciences, Hawassa University, Ethiopia. \\ ${ }^{3}$ BSc., MSc.,In Medical Parasitology, Department of Medical Laboratory Sciences, College of Medicine \\ and Health Sciences, Hawassa University, Ethiopia. \\ ${ }^{4}$ BSc., MSc., In Medical Microbiology, Department of Medical Laboratory Sciences, College of Medicine \\ and Health Sciences, Dire Dawa University, Dire Dawa, Ethiopia.
}

Article Info: Received 20 January 2019; Accepted 14 February. 2019

Cite this article as: Wubete, M., Mohammed, M., Mengistu, M., \& Mekonnen, R. (2019). PREVALENCE OF SYPHILIS AMONG PREGNANT WOMEN ATTENDING ANTENATAL CLINIC OF BULCHANA HEALTH CENTER, SHASHEMENE, WEST ARSI ZONE, ETHIOPIA. International Journal of Medical and Biomedical Studies, 3(2).

DOI: https://doi.org/10.32553/ijmbs.v3i2.90

Address for Correspondence: Robel Mekonnen, BSc., MSc., In Medical Microbiology, Department of Medical Laboratory Sciences, College of Medicine and Health Sciences, Dire Dawa University, Dire Dawa, Ethiopia.

Conflict of interest: No conflict of interest.

\section{Abstract}

Background: Syphilis is an important cause of morbidity and mortality in pregnancy and it is one of the greatest public health challenges. It is responsible for up to $30 \%$ of perinatal mortality. Ethiopia is one of the countries in which sexually transmitted infection (STIs) are highly prevalent. However, the data needed to present a realistic picture of the prevalence of syphilis are limited.

Objective: To determine the prevalence of syphilis among pregnant mothers attending Antenatal Clinic of Bulchana health center, Shashemene, West Arsi Zone, Ethiopia, 2017.

Materials and Methods: Health institution based retrospective cross sectional study was conducted from April 01 to June 01, 2017 among women attended Antenatal Clinic of Bulchana health center during the period of 2014 to 2016. Non probability sampling technique was used to recruit study participants. A total of 4346 pregnant women were included in this study. Data was captured by reviewing and observation of a recorded data at the ANC registration book using pre-tested structured check list. Data were entered and cleaned using EpiData version 3.0. and analyzed by SPSS version 21. Binary logistic regression was used and significant variables were further adjusted using multivariate analysis. A $p$-value $<0.05$ was considered as level of significance.

Result: In this study the prevalence of syphilis among pregnant women found to be $1.1 \% 95 \% \mathrm{Cl}[0.8$ 1.3]. Relatively high prevalence of syphilis 15(1.5\%) was observed among age group of 15-19 years. The prevalence of syphilis was high among pregnant women living in rural area 35(1.1\%). Being rural dwellers and those with multiple pregnancy, 2-4 and more than 5 pregnancies showed significant 
association with prevalence of syphilis infection. [(OR=0.68, 95\% Cl $(5.33,17.32), P=0.04)],[(O R=10$, $95 \% \mathrm{Cl}(1.36,74.42), \mathrm{p}=0.02]$ and $[(\mathrm{OR}=2.18,95 \% \mathrm{Cl}(1.20,3.95), \mathrm{p}=0.01]$, respectively.

Conclusion: The observed seroprevalence of syphilis among women attending ANC still mandates effective health education campaigns to elucidate the risk factors and prevention of syphilis not only to pregnant women but also the public at large. Strengthening service delivery at the provider and facility level to improve prompt access to effective syphilis diagnostic and treatment services is mandatory.

Keywords: syphilis, pregnant women, ANC, Shashemene Ethiopia.

\section{Introduction:}

Syphilis is a sexually transmitted infection (STI) caused by the spirochete Treponema palladium [1]. Generally considered to be sexually transmitted disease, this infection can also be transmitted in utero and rarely by blood transfusion or non-sexual contact [2]. It is of particular concern during pregnancy because of the risk of trans-placental infection of the fetus due to hematogeneous spread from an infected mother. Even though Treponema palladium can cross the placenta and infect the fetus in the early gestation, the risk of adverse pregnancy outcomes increase with age [3].

Syphilis is an important cause of morbidity and mortality in pregnancy and it is one of the greatest public health challenges. It is responsible for up to $30 \%$ of perinatal mortality [4]. Global and regional estimates of the burden of maternal syphilis and related adverse outcomes for estimates suggest that, nearly 1.4 million pregnant women globally are infected with probable active syphilis accounting for approximately $1 \%$ of all pregnancies worldwide $[5,6]$. Africa and Asia are regions with high disease burden, representing $39.3 \%$ and $44.3 \%$ of the global estimate, respectively [6].

Syphilis is common in Africa and without appropriate treatment $5-8 \%$ of all pregnancies may have an adverse outcome [7]. Untreated maternal syphilis leads to a significant reproductive health burden and contributes to syphilis-associated adverse pregnancy outcomes, including stillbirth and late fetal loss, neonatal death, premature and low birth weight infants, as well as congenital syphilis [1, 8]. Adverse pregnancy outcomes are influenced by gestational age, stage of maternal syphilis, and immunological response of the fetus [3].

Congenital syphilis pose a significant challenge especially because infants may be still born, asymptomatic at birth but nonetheless infected, or present with a highly variable clinical picture, thereby precluding easy clinical diagnosis. The risk of congenital syphilis in untreated or inadequate treated mothers $(4 \%)$ is one reason why $40 \%$ of these pregnancies end in fetal loss or perinatal death $[4,9]$.

Although antenatal (ANC) syphilis screening has proven to be cheap and effective; syphilis during pregnancy continues to be a substantial problem in resource-poor settings. The rapid plasma reagin (RPR) $18 \mathrm{~mm}$ circle card test for syphilis is used as a screening test in many ANC clinic and health facilities in the developing world. Although it is easy to perform and inexpensive it may be difficult to interpret and requires training of health personnel to ensure testing is carried out and results are read correctly [10].

Ethiopia is one of the countries in which STIs are highly prevalent. However, the data needed to present a realistic picture on the magnitude of syphilis are limited. Moreover, few studies conducted in Ethiopia reported different prevalence across the country. For instance, syphilis was accounted for $2.7 \%$ and $2.2 \%$ of the STDs reported in the years 2008 and 2010, respectively [11]. A study conducted in Addis Ababa revealed that $2.9 \%$ mothers were positive for Venereal Disease Research Laboratory (VDRL) [12]. In 2014, a study done in Gonder teaching hospital reported $2.9 \%$ prevalence of syphilis among pregnant women using RPR/ TPHA test [13]. However, in 2007, the seroprevalence of 
syphilis in the same hospital was documented as $1 \%$ [14]. On the other hand, high prevalence of syphilis (12.1\%) was reported in Jimma teaching Hospitals in 2002 [15].

To understand the reasons why a curable disease continues to survive, it is important to understand its prevalence in a specific region, Shashemene, which is one of the largest cities in Ethiopia, comprising a population having different customs, cultures, nations and nationalities. However, there is limited information and no locally generated data on the prevalence syphilis in the study area. It is important to understand prevalence of syphilis among pregnant mothers in our study area and ultimately what factors are contributing for occurrence of such a disease. Therefore, this study aim to determine the prevalence of syphilis and associated risk factors among pregnant women who attended ANC clinics.

\section{Methods and Materials}

\section{Study Area}

This study was conducted in Bulchana health center, Shashemene, West Arsi zone. Shashemene town was established in 1911. It is located at $7^{\circ}$ North and $38^{\circ}-37^{\circ}$ East longitude in the main Ethiopia Rift Valley at a distance of 250 $\mathrm{km}$ South of Addis Ababa and $25 \mathrm{Km}$ from Hawassa, the capital city of South Nation Nationalities and Peoples Region (SNNPR). The average elevation of the town is about $1940 \mathrm{~m}$ above sea level with $3 \%$ slope along river streams crossing the city. The temperature level ranges from $12-28^{\circ} \mathrm{C}$ and yearly rainfall varies from 1,500-2,000mm.

Based on figures published by the Central Statistical Agency in 2007, the total population of the City is 339,981 , of whom 171,161 are men and 168,820 , are women. 105,939 or $31.16 \%$ of its population are urban dwellers [16].

Bulchana health center is one of the health rendering services in shashemene town. It is established in 2009 and serves around 50,500 populations. This health center provides different services such as antenatal care services, family planning, delivery, counseling's services, infant and adult OPD and laboratory services.

\section{Study Design and Period}

A health institution based retrospective cross sectional study was conducted among pregnant women attended ANC of Bulchana health center in the period of January 2014 to December 2016.

\section{Population}

\section{Source Population}

All pregnant women who attended ANC service in Bulchana health center from January 2014 to December 2016.

\section{Study Population}

Pregnant women who had visited ANC clinic and have tested for syphilis and recorded on the registration book during January 2014 to December 2016.

\section{Inclusion and Exclusion Criteria}

Pregnant women who have full demographic data and who had test result for Rapid Plasma reagin (RPR) test were included. Whereas pregnant women with incomplete recorded demographic data and RPR test were excluded.

\section{Sample size Determination and Sampling Technique}

A medical record of 4346 pregnant women who attended ANC of Bulchana health center were reviewed. Non probability sampling techniques were used to enroll the study participants from January 2014 to December 2016.

\section{Study Variables}

Prevalence of syphilis was the dependent variable

Independent variables include socio demographic characteristics like age, residence, educational level, marital status. Other factors such as number of pregnancy, history of STI.

\section{Data Collection Procedures}

Secondary data were collected using structured pre tested check list. The tally sheet was filled with great care and the quality maintained by 
performing the tally by two independent data collectors on a separate sheet. The discordant number was cross checked again. Ambiguity during the collection process from the log book was excluded from the analysis. But, to overcome the ambiguity the senior professional in the $\mathrm{MCH}$ department had assisted during the data collection process for clarification of unreadable/illegible data on the log book.

\section{Data Quality Control}

Training was given for data collectors regarding the aim of study, data collection tool and procedure before the actual data collection. During data collection seropositive and seronegative recorded data of pregnant women who enrolled in $\mathrm{MCH}$ department was crosschecked against laboratory log book. Moreover, the check list was pre-tested on $5 \%$ of sample size among recorded data before actual data collection. Four laboratory professionals were recruited to collect the data and two senior health professionals were recruited as supervisors. Data quality was ensured during collection, coding, entry and analysis by investigators and supervisors. The collected data were checked on daily basis for completeness and consistency.

\section{Data Processing and Analysis}

Data were sorted, coded and entered in to computer using Epi Data version 3.02 after checked for completeness and accuracy. Then data were exported to SPSS database program version 21.0 for further analysis. Univariate analysis and appropriate graphic presentations was used for describing and presenting the data. Bivariate analysis of demographic and other factors associated with syphilis infection was done and to ascertain the association variables found to be significant $(p<0.25)$ in the bivariate analyses were used to construct a multivariate models. Adjusted odds ratio along with 95\% Confidence Interval $(\mathrm{Cl})$ was estimated to identify factors associated with prevalence of syphilis using multivariate logistic regression analysis. Variables with $p$-value $<0.05$ was declared as statistically significant.

\section{Results}

\section{Socio Demographic Characteristics of the Respondents}

A total of 4346 pregnant women were examined for sero-positivity of syphilis between January 2014 and December 2016. As displayed in Table 1 below, age of the participated women ranged from 15 to 44 years with a mean of 29.5 years. Similarly, majority $1271(29.2 \%)$ of women attending ANC were between 20-24 years of age, followed by $1159(26.7 \%)$ of pregnant women found in age group of 25-29 years. Three thousand and two hundred sixteen (74\%) pregnant women were urban dwellers while the rest 1130 (26\%) were from rural area.

Out of the total 4346 pregnant women attending ANC between 2014 and 2016, 4237(97.5\%) of them were married and only $98(2.3 \%)$ were unmarried. The rests $8(0.2 \%)$ and $3(0.1 \%)$ were divorced and windowed, respectively. On the other hand, 1495(34.4\%) were pregnant for the first time while $2289(52.70 \%)$ of the study subjects had 2-4 pregnancies.

Figure - 1 displayed ANC service utilization and tests for syphilis by the study period, the high number of ANC attendants were in the year 2015, followed by in the year 2016 and 2014, which accounts 1502(34.6\%) and 1483 (34.1\%), respectively.

\section{Prevalence of syphilis}

As shown in Table -2 below, out of 4346 total pregnant women, $46(1.1 \%) 95 \% \mathrm{Cl}$ [0.8-1.3] were screened as seropositive for syphilis. Relatively high seroprevalence of syphilis $15(1.5 \%)$ were observed among pregnant women of age group $15-19$ years old, followed by $14(1.2 \%)$ in the age group of 25-29 years. However, seropositive for syphilis was not detected among pregnant women in the age group of $40-44$ years. About $35(1.1 \%)$ of syphilis infection was detected among pregnant women from urban areas. Higher prevalence of syphilis infection was documented in 2015, followed by 2016 which accounts $20(1.3 \%)$ and $15(1.0 \%)$, respectively. 
Among the total RPR reactive pregnant women attending ANC, 45(1.1\%) were married and $1(1.0 \%)$ were unmarried. Similarly, 19(1.3\%) seropositive women had pregnant for the first time and $26(1.1 \%)$ had 2 to 4 pregnancies.

On multivariate analysis of the dependent and independent variables, residence and number of pregnancy found to be significantly association with seroprevalence of syphilis. Accordingly pregnant women from rural areas had 32\% less likely to be infected with syphilis compared with those from urban area $(P=0.04, O R=0.68$, $95 \% \mathrm{Cl}[5.33,17.32])$. Similarly, pregnant women with multiple pregnancy $(>5)$ had 10 times greater risk of syphilis infection compared with those having single pregnancy $(P=0.02 \mathrm{OR}=10$, 95\% Cl [1.4-74]) (Table -2).

Table 1: Socio-demographic and obstetrical characteristics of pregnant women attended Bulchana Health Center during the period of January 2014 to December $2016(n=4346)$, Shashemene, West Arsi Zone, Ethiopia

\begin{tabular}{|c|c|c|c|}
\hline Characteristics & Catagory & Frequency(n) & Percent (\%) \\
\hline \multirow[t]{7}{*}{ Age group } & $15-19$ & 1012 & 23.3 \\
\hline & $20-24$ & 1271 & 29.2 \\
\hline & $25-29$ & 1159 & 26.7 \\
\hline & $30-34$ & 543 & 12.5 \\
\hline & $35-39$ & 240 & 5.5 \\
\hline & $40-44$ & 121 & 2.8 \\
\hline & Total & 4346 & 100.0 \\
\hline \multirow[t]{3}{*}{ Residence } & Urban & 3216 & 74.0 \\
\hline & Rural & 1130 & 26.0 \\
\hline & Total & 4346 & 100.0 \\
\hline \multirow[t]{5}{*}{ Marital status } & Married & 4237 & 97.5 \\
\hline & Unmarried & 98 & 2.3 \\
\hline & Divorced & 8 & 0.2 \\
\hline & Widowed & 3 & 0.1 \\
\hline & Total & 4346 & 100.0 \\
\hline \multirow{4}{*}{$\begin{array}{ll}\text { Number } & \text { of } \\
\text { pregnancies }\end{array}$} & 1 & 1495 & 34.4 \\
\hline & $2-4$ & 2289 & 52.7 \\
\hline & $>5$ & 562 & 12.9 \\
\hline & Total & 4346 & 100.0 \\
\hline
\end{tabular}


Table 2: Multivariate logistic regression analysis of Risk factors associated with syphilis among pregnant women who attended in Bulchana Health Center during the period of January 2014Decebmer 2016 ( $n=4346)$, Shashemene, West Arsi Zone, Ethiopia

\begin{tabular}{|c|c|c|c|c|c|c|}
\hline \multirow{2}{*}{\multicolumn{2}{|c|}{ Risk factors }} & \multicolumn{2}{|c|}{ Result of RPR } & \multirow{3}{*}{$P$ value } & \multirow{3}{*}{$\begin{array}{l}\text { AOR } \\
\\
\mathrm{R} \\
\end{array}$} & \multirow{3}{*}{$95 \% \mathrm{Cl}$} \\
\hline & & \multirow{2}{*}{$\begin{array}{l}\text { Reactive } \\
15(1.5)\end{array}$} & \multirow{2}{*}{$\begin{array}{l}\text { Non-reactive } \\
996(98.5) \\
\end{array}$} & & & \\
\hline Age group & $15-19$ & & & & & \\
\hline & $20-24$ & $11(0.9)$ & $1260(99.1)$ & 0.14 & 1.80 & $0.81-3.92$ \\
\hline & $25-29$ & $14(1.2)$ & $1145(98.8)$ & 0.54 & 1.3 & $0.60-2.62$ \\
\hline & $30-34$ & $4(0.7)$ & $540(93.3)$ & 0.19 & 2.1 & $0.69-6.37$ \\
\hline & $35-39$ & $2(0.8)$ & $238(99.2)$ & 0.45 & 1.770 & $0.40-7.81$ \\
\hline & $40-44$ & 0 & $121(100.0)$ & 0.99 & & \\
\hline \multirow[t]{2}{*}{ Residence } & Urban & $35(1.1)$ & $3181(98.9)$ & & $\mathrm{R}$ & \\
\hline & Rural & $11(1.0)$ & $1119(99)$ & 0.04 & 0.68 & $5.33-17.32$ \\
\hline \multirow[t]{4}{*}{ Marital status } & Married & $45(1.1)$ & $4192(98.9)$ & & $\mathrm{R}$ & \\
\hline & Unmarried & $1(1.0)$ & $97(99)$ & 0.89 & 1.15 & $0.15-8.49$ \\
\hline & Divorced & 0 & $8(100.0)$ & 0.99 & & \\
\hline & Widowed & 0 & $3(100.0)$ & 0.99 & & \\
\hline \multirow[t]{3}{*}{ Study year } & 2014 & $11(0.8)$ & $1353(99.2)$ & & $\mathrm{R}$ & \\
\hline & 2015 & $20(1.3)$ & $1480(98.7)$ & 0.81 & 0.60 & $0.28-1.26$ \\
\hline & 2016 & $15(1.0)$ & 1468(99.0) & 0.57 & 0.79 & $0.36-1.74$ \\
\hline \multirow{3}{*}{$\begin{array}{l}\text { Number of } \\
\text { pregnancies }\end{array}$} & 1 & $19(1.3)$ & $1476(98.7)$ & & $\mathrm{R}$ & \\
\hline & $2-4$ & $26(1.1)$ & $2263(98.9)$ & 0.01 & 2.18 & $1.20-3.95$ \\
\hline & $>5$ & $1(0.2)$ & $561(99.8)$ & 0.02 & 10.0 & $1.36-74.42$ \\
\hline
\end{tabular}

R (Reference), * (“p<0.05”) Statistically significant.

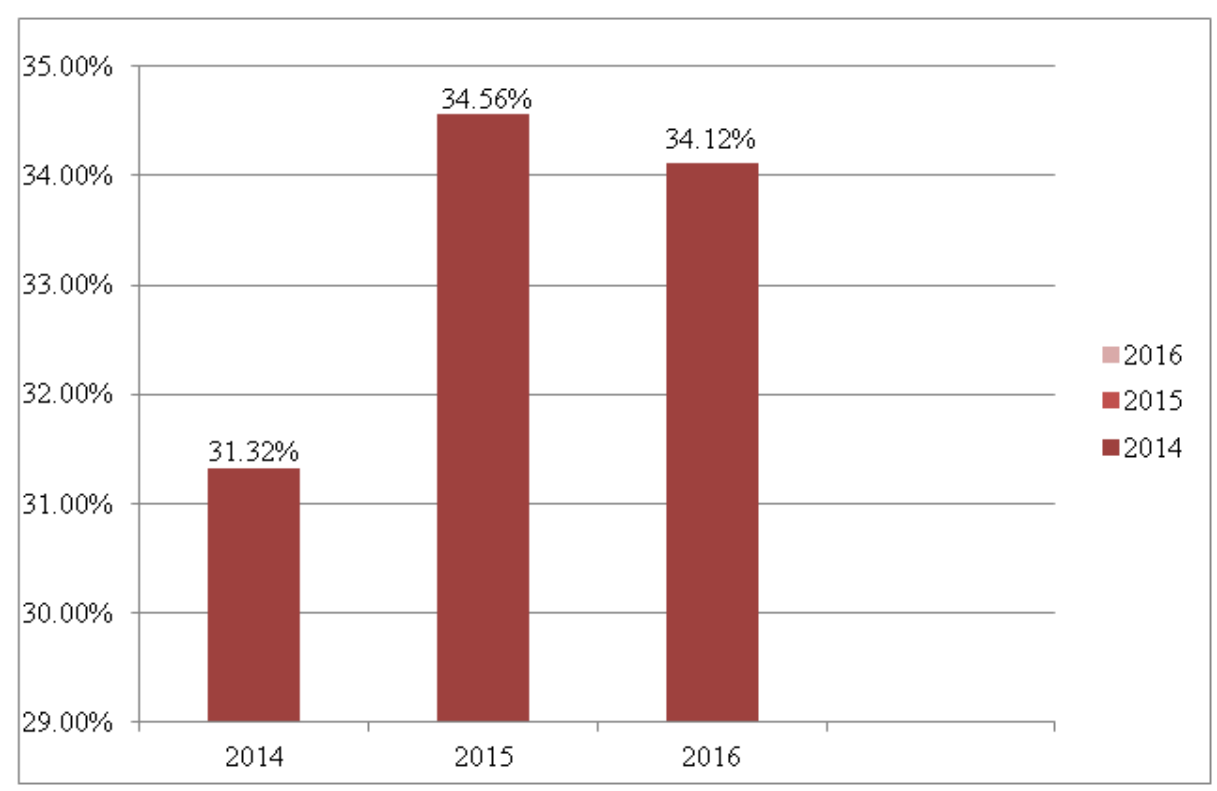

Figure 1: Year distribution of pregnant women attending ANC in Bulchana health center, in the period of January 2014 to December 2016 


\section{Discussion}

Syphilis still affects high proportion of pregnant women worldwide, causing serious health problems and even death to their babies, yet this infection could be prevented by early testing and treatment [17]. In sub-Saharan Africa a prevalence of syphilis among pregnant women range from 2.5 to $18 \%$, with the highest prevalence in the age group $35-49$ years $[18,19]$. According to 2007 WHO report syphilis infection rates in pregnant women in Africa ranges from $3 \%$ to15 \% [20].

In the present study, the prevalence of syphilis among pregnant women was found to be $1.1 \%$. This figure is slightly higher than the seroprevalence of syphilis reported in Mexico (0.26\%) [21], Brazil (1.06\%) [22], Pakistan (0.9\%) [23] India (1 to $0.9 \%$ ) [24]. On the other hand, it is lower than previous reports from Bangladesh (2.96\%) [25], Nigeria (1.9\%) [26], Tanzania (2.5\%) [27], Madagascar (3\%) [28], Southern Sudan (22.1\%) [29], Addis Ababa (2.9\%) [12], Gonder teaching hospital in 2014 (2.9\%) [13], Gonder teaching hospital in 2007 (1\%) [14], and Jimma in 2002 (12.1\%) [15].

The low prevalence rate found in the present study compared with previous studies in Ethiopia may indicate a change in the prevalence of syphilis in Ethiopia over the last decade. This might be attributed by difference in socioeconomic status or geographical location as well as study period or study design. Another possible explanations might be due to use of different diagnostics test kits with high quality of sensitive by the previous studies such as EIA and TPHA. That means RPR is the only test kit used for screening syphilis in the study area. Because of high false negativity of RPR test that occur both in early primary cases [30] and among patients with secondary syphilis, as a result of prozone reactions [31], may limit the sensitivity of the test thereby affecting the prevalence of syphilis in the study area. Hence, the appropriateness of syphilis screening using RPR testing in ANC clinics and health centres should be questioned if there is a low prevalence in the population, conditions for testing are poor, and resources are limited [10]. Moreover, in many developing country, settings where the RPR test would be useful as a screening test, such as ANC clinics, quality control procedures are suboptimal or lacking entirely and the rate of false positives and false negatives associated with the use of the test (and consequent overtreatment or undertreatment for syphilis) may be higher under operational conditions than that anticipated from research reports[10].

In the present study, different seroprevalence of syphilis was observed across different age group but the difference was not statistically significant $(P=0.54)$. Women in the age group of $15-19$ years had high seropositivity (1.5\%). This finding was consistent with the report in Jimma [15], but the report from Nigeria and Gonder teaching hospital revealed that the most affected age groups were 20-24 years old [13, 27]. Moreover women with age group of 30-34 years and those above 40 years had low seropositivity which is in agreement with the report in South Africa and in Addis Ababa [12]. The variation of syphilis prevalence with different age group may be explained as a reflection of the differences in sexual practices, such as a numbers of sexual partners. Hence, these findings highlighted that young pregnant mothers are susceptible to syphilis infection. This may be due to unsafe sexual practices like unprotected sex.

We found a high prevalence of syphilis among pregnant women coming from urban area (1.1\%) as compared to those coming from rural areas $(1 \%)$ and this was statistically significant $(P=0.04$, $\mathrm{OR}=0.68,95 \% \mathrm{Cl}[5.33,17.32])$. In contrast to our finding, a similar study done in Tanzania which revealed high seroposetivity (3.13\%) of syphilis among pregnant women attending rural clinics as compared to urban clinics (3.01\%) [28]. This might be due to inaccessibility of ANC service and/ or lack of awareness of the services by the rural dwellings. Our findings of $1.1 \%$ seropositivity of syphilis in urban pregnant women population was much lower than the previous 
study reported from Gonder teaching hospital, which was $3.2 \%$ [13].

In this study, different rate of syphilis was found at different periods. For instance, it was $0.8 \%$ in year $2014,1.3 \%$ in year 2015 , and $1.0 \%$ in year 2016. However, the observed difference was not significant $(\mathrm{P}=0.38)$.

The difference of seroposetivity of syphilis among numbers of pregnancy was statistically significant. Accordingly, pregnant women having 2 to 4 pregnancies and more than 5 pregnancies were more likely to be infected $(P=0.01, O R=2.18$ , 95\% Cl[1.20, 3.95]) and $(P=0.02, O R=10.0$, $95 \% \mathrm{Cl}[1.36-74.42])$ which is in consistent with study done in Tanzania $(P<0.05, O R=2.48,95 \%$ $\mathrm{Cl}[2.00-3.08])$ [28].

In this study we also observed relatively high seroprevalence rate of syphilis among married pregnant women (1.1\%). However, the distribution of syphilis among marital status was not significant( $P>0.98)$.

\section{Conclusion}

It can be concluded that the present study revealed comparatively low seroprevalence of syphilis among pregnant women attending ANC tested by RPR in the study area. The low seroposetivity rate found in this study may demonstrate the improvement of ANC service at different levels of health care. On the other hand, false negatives that occur both in early primary cases and in patients with secondary syphilis, may limit the sensitivity of RPR test. This indirectly shows the possibility of missed cases and failure in early diagnosis and treatment of syphilis infection thereby increasing adverse pregnancy outcomes associated with syphilis infection.

Although different seropositivity of syphilis found among different groups of the study participants, pregnant women from rural communities and those having multiple pregnancies were significantly associated with syphilis infection. Hence, an increase in numbers of pregnancy (gravidity) was significantly associated with increased risk of syphilis infection.

\section{Recommendations}

$\checkmark$ Effective health education campaigns and condom promotion activities will be needed to elucidate the risk factors and prevention of syphilis not only to pregnant women but also the public at large.

$\checkmark$ Strengthening service delivery at the provider and facility level to improve prompt access to effective syphilis infection diagnostic and treatment services.

$\checkmark$ Strengthening routine screening for syphilis in antenatal to further reduce the disease among pregnant women.

Appropriate strategies should be designed for prevention and control of STD in women of reproductive age groups and the general population.

Further research should be conducted in order to explore the risk factors that aggravate the incidence of syphilis in the community.

\section{Acknowledgements}

The authors are grateful to all participated pregnant women and all the staff of Bulchana Health center for their cooperation in undertaking this research. We also extend our gratitude to data collectors and other research team.

\section{Authors' contributions}

MW was major contributor designed the study, participated in data collection, analysis, interpretation and write-up. $\mathrm{MM}$ and $\mathrm{MM}$ participated in the data collection, analysis, and interpretation. RM participated in the analysis, interpretation and write-up, drafted the manuscript and critically revised the manuscript. All authors read and approved the final manuscript.

\section{Funding}

The present study did not received financial support from any sources. 


\section{Ethical approval}

Letter was obtained from Hawassa University, College of medicine and health science, Community Based Education (CBE) office. A formal letter was submitted to all the concerned bodies in the study area to obtain their cooperation in facilitating the study. The data collectors were briefed about the objective, benefit, confidentiality and risks of the study prior to data collection.

\section{Reference}

1. Centers for Disease Control and Prevention. (2015). Sexually Transmitted Disease Surveillance,. Available at http://www.cdc. gov/std/stats15/std-surveillance-2015, $18 \underline{251}$ -259 .

2. Goh BT. (2005). Syphilis in adults. Sex transm Infect.; 81: 448-52.

3. Salooje H, Velaphi S, Goga Y, Afadapa N, Steen R, Lincetto O. (2004). The prevention and management of congenital syphilis: an an overview and recommendations. Bull World Health Organ; 82(6):424-430.

4. DelportSD, Rothberg AD (1992). Congential syphilis now a notifiable disease .South African Medical Journal;81;288-289.

5. World Health Organization (2014). Global prevalence and incidence of selected curable sexually transmitted infections. WHO; 21:27

6. World Health Organization (2012). Investment case for eliminating mother-tochild transmission of syphilis, Geneva.12; ISBN 9789241504348.

7. Schultz KF, cates $W$ Jr, O Mara PR (1987). Pregnancy loss, infat death and suffering: legancy of syphilis and gonorrhea in Africa .Genitourinary Medicine; 63;320-325.

8. Gomez, GB; Kamb,ML, Newman, LM; Mark,J;Brouetet,N; Hawkes;SJ. (2013). Untreated maternal syphilis and adverse outcomes of pregnancy: a systematic review and meta-analysis, Bull World Health Organ., 91, pp. 217-226.

9. CDC congenital syphilis, Net York city. 20012003. MMWR. 2004;38:825-9.
10. B West, G Walraven, L Morison, J Brouwers, $R$ Bailey (2002). Performance of the rapid plasma reagin and the rapid syphilis screening tests in the diagnosis of syphilis in field conditions in rural Africa. Sex Transm Infect; $78: 282 \pm 285$

11. World health Organization (2012). Base line report on global sexually transmitted infection surveillance, ISBN 978924150589 5,50-58.

12. E.Kebede, and B.Chamiso, (2011). prevalence of syphilis in pregnancy in Addis Ababa. J East African medical journal Vol.77, No.4, April 2000. 2012 Mar; 53(3):268-9. doi: 10.1016/j.jcv.2011.12.004.

13. Abate A. (2014). A three year Retrospective study on seroprevalence of syphilisamong pregnant women at Gonder University Teaching Hospital, Ethiopia, African Health science; vol 14,No.1.

14. Mulu A, Kassu A,Tessema B,Yismaw G, Tiruneh M,Moges $F$, WondimkunY, Nishikawa T, Ota F. (2007). Seroprevalence of syphilis during pregnancy in a teaching hospital in northwest Ethiopia, Jpn. J. Infect. Dis., 60 (4), 193-195.

15. Mekonnen Z,Tegbaru B, Meless H . (2002). Seroprevalence of syphilis and HIV-1 among pregnant women attending ANC clinics in Jimma Hospital Southern Ethiopia.Ethio J health sciences ;12(2):81-89.

16. Centeral Stastical Authority, (2007). population and housing census of Ethiopia, Administrativereport,April 2012.

17. Lori Newman, Mary Kamb, Sarah Hawkes, Gabriela Gomez, Lale Say, Armando Seuc, NathalieBroutet. Global estimates of syphilis in pregnancy and associated adverse outcomes: analysis of multinational antenatal surveillance data. PLoS Med. 2013; 10(2):e1001396

18. Mullick $S$, Watson-Jones $D$, Beksinnska $M$, and Mabey D. Sexually transmitted infections pregnancy: prevalence, impact on pregnancy outcomes, and approach to treatment in developing countries. SexTransInfect2005; 8:294-302. 
19. Mehmet GencWilliamJLedgerSyphilisin pregnancy sex transm Inf2000; 76:73-79.

20. Schmid G. Economic and programmatic aspects of congenital syphilis prevention. Bull WHO 2004; 82(6):402-409

21. Yanez Alvarez L, Conde Gonzalez CJ, Uribesalas FJ, Sanchez-Alman MA ,Maternal / child seroprevalence of antibodies against Treponema palladium at four general hospitals in the state of Morelos, Mexico,Arch med Res, 2012 oct 43(7); 571-7.

22. Rosa, Maria Soares Madeira Domingues, Maria do carmo Leal, Paolo Roberto B, Souza J. Prevalence of syphilis in pregnancy and prenatal syphilis testing in Brazil: Birth in Brazil study,Rev. Saude publica vol. 48 no 5 sao Paulo oct. 2014.

23. Sa Shah, S Krstensen, MA Memon G Usman, A Ghazi, R John, SH Vermund. Prevalence of syphilis among antenatal clinic attendees in Karachi: Imperative to begin universal screening in Pakistan,J Pak Med Assoc. 2011 Oct; 61(10): 993-999.

24. Nazish F, AbidaMalik P, Anwar K, Sana A ,Haris M. Seroprevalence of infection among patients attending Antenatal care Sexually Transmitted Disease clinics: Observation from a Tertiary care Hospital of Northern India , American Journal of InternalMedicine. Vol,2,No.1,2014, pp,69, doi:10.11648.

25. Nessa A, Islam F, Khanam A, Sultana N, Rashid $\mathrm{MH}$. Seroprevalence of Treponema palladium antibody among pregnant population of Bangladsh. J Obstet Gynacol Res. 2011; Nov;37(11): 1625-30.

26. Mustefa Alhaji Isa. prevalence of syphilis among pregnant women attending antenatal state specialist Hospital Maiduguri ,Borno,Nigeria.Centeral Africa Medical Journal,2014;7:36

27. Joel M, Mathias I, James J ,Bonita K. Prevalence of HIV and syphilis infection among pregnant women attending, antenatal clinics in Tanzania,2015 BMC Public Health 2015 15:501

28. Schwarz NG Girman M, Hagen RM, Poppert $S$, Crusius . Serological survey of HIV and syphilis in pregnant women in Madagascar, Syphilis sero-positivity among pregnant women attending public antenatal clinics: Southern African Journal of Infectious Diseases , Vol 31, 2016 - Issue 3)

29. Emmuanuel SK, Lado M, Amwayi $S$, Abade AM, Qundo JO, Ongus JR, . Syphilis among pregnant women in Juba, Southern Sudan.East African Med J 2010,may 87(5):192-8.

30. Larsen S, Steiner B, Rudolph A.Laboratory diagnosis and interpretation of tests for syphilis. Clin Microbiol Rev 1995;8:1 21 .

31. Berkowitz K, Baxi L, Fox HE. False negative syphilis screening: thet prozone phenomenon, non-immunehydrops, and diagnosis of syphilis during pregnancy.Am J Obstet Gynecol 1990;163:975 \pm 7 